\title{
CALCULATION OF CAUCHY-TYPE INTEGRALS NEAR CONTOURS IN DIRECT AND INVERSE ELASTIC PROBLEMS
}

\author{
ALEXANDER N. GALYBIN \\ Schmidt Institute of Physics of the Earth (IPE RAS), Russia
}

\begin{abstract}
This study presents an approach for the calculation of Cauchy-type integrals at points located near contours. It is evident that the kernel of a Cauchy integral becomes close to singular as soon as one intends to calculate the value of the integral close to the contour. As a result, more nodes in a quadrature formula are needed, in order to reach acceptable accuracy in the calculations. This problem is faced in standard formulations when analysing stress-strain states after obtaining numerical solutions of certain singular integral equations; as well as in non-classical formulations, where the data close to the contour are used as input. On the other hand, one can employ, for the contour points, the Plemelj-Sokhotski formulas, assuming calculation of the singular integral is followed by addition of a known non-integral term. In this study, we use expansions into power series to calculate stress characteristics at points near the contours, suggest an algorithm, and numerically analyse two cases that are relevant to direct and inverse formulations in plane elasticity.
\end{abstract}

Keywords: plane elasticity, Cauchy integrals, singular integral equations, inverse problem, mathematical optimisation.

\section{INTRODUCTION}

This study aims to develop numerical techniques for the calculation of complex potentials near contours. It is well known that the general solution of a plane elastic problem is expressed via two independent complex potentials [1]. These can be presented by Cauchytype integrals with unknown densities, as follows:

$$
\Phi(z)=\frac{1}{2 \pi i} \int_{\Gamma} \frac{g(t)}{t-z} d t, \quad \Psi(z)=\frac{1}{2 \pi i} \int_{\Gamma} \frac{h(t)}{t-z} d t .
$$

Here, $\Gamma$ is a closed or open contour (or set of contours), and the densities $g(t)$ and $h(t)$ are not independent of each other. They are found by solving certain singular integral equations corresponding to a particular boundary value problem under consideration.

The method of mechanical quadratures [2] is often used to solve a system of complex singular integral equations (CSIE), because of its high efficiency and simplicity in programming. The sought density is found at the collocation points that depend on the quadrature formulas used, usually presenting the roots of the Chebyshev polynomials. Interpolation at other contour points can be made by different methods, such as linearly piecewise, cubic splines or by interpolation polynomials. It is worth noting that the accuracy of the interpolated solution is always not as good as the accuracy of the solution at the collocation points; that is, $2 n$ where $n$ is the order of the Chebyshev polynomials used in approximation of the sought density. The contour values of the complex potentials are found by the Plemelj-Sokhotski formulas:

$$
2 \Phi^{ \pm}(\zeta)= \pm g(\zeta)+\mathbf{S}(g), \quad \zeta \in \Gamma
$$


where $\mathbf{S}(\mathrm{g})$ is the singular integral below:

$$
\mathbf{S}(g)=\frac{1}{\pi \mathrm{i}} \int_{\Gamma} \frac{g(t)}{t-\zeta} d t, \quad \zeta \in \Gamma .
$$

Numerical methods for the calculation of singular integrals (3) are well developed. For instance, if $\Gamma$ is an open interval $(-1,1)$, then for unbounded solutions at the ends, the following quadrature formula can be used [2]:

$$
\int_{-1}^{1} \frac{u(t)}{\sqrt{1-t^{2}}(t-x)} d t=\frac{\pi}{n} \sum_{k=1}^{n} \frac{u\left(\xi_{k}\right)}{\xi_{k}-x}+\pi u(x) \frac{U_{n-1}(x)}{T_{n}(x)}, \quad|x|<1 .
$$

Here $T_{n}(x)=\cos (n \arccos x), \quad U_{n-1}(x)=\sin (n \arccos x) / \sqrt{1-x^{2}}$ are the Chebyshev polynomials of the first and second order, respectively; while the nodes $\xi_{k}$ are the roots of $T_{n}(x)$, such as: $\xi_{k}=\cos \frac{2 k-1}{2 n} \pi, \quad k=1, \ldots n$. It is evident that the second term on the righthand side of eqn (4) disappears at the roots of $U_{n-1}(x)$, i.e., at the points $\eta_{m}=\cos \frac{m \pi}{n}, \quad m=1, \ldots n-1$. Therefore, eqn (4) becomes:

$$
\int_{-1}^{1} \frac{u(t)}{\sqrt{1-t^{2}}\left(t-\eta_{m}\right)} d t=\frac{\pi}{n} \sum_{k=1}^{n} \frac{u\left(\xi_{k}\right)}{\xi_{k}-\eta_{m}}
$$

This coincides with the standard quadrature formula for regular integrals. This equation is exact, if $u(t)$ is a polynomial of up to $2 n$ degree.

Consider the Cauchy-type integral in eqn (1) and apply the same quadrature formula to it, which results in:

$$
\int_{-1}^{1} \frac{u(t)}{\sqrt{1-t^{2}}(t-z)} d t=\int_{-1}^{1} \frac{(t-x)-i y}{(t-x)^{2}+y^{2}} \frac{u(t)}{\sqrt{1-t^{2}}} d t=\frac{\pi}{n} \sum_{k=1}^{n} \frac{\left(\xi_{k}-x\right)-i y}{\left(\xi_{k}-x\right)^{2}+y^{2}} u\left(\xi_{k}\right), \quad|x|<1 .
$$

This equation is exact if the integrand (without the weight) is a polynomial of up to $2 n-1$ degree. We can see that for small $y$ the denominator in eqn (6) becomes close to singular, and so cannot be accurately approximated by the polynomials. In practice, it means that for acceptable accuracy, one would need to use approximations using polynomials of large degrees; thus, a large number of nodes and collocation points would be required. It can be estimated that for the values of $y$ smaller than the minimum difference of $\xi_{k}-\eta_{m}$ there would be strongly degraded accuracy of eqn (6).

We aimed to develop simple algorithms that do not require the use of a large number of nodes for the calculation of integrals in eqn (1), specifically for the points located close to the contours. Our main idea was to use the properties of holomorphic functions and Taylor's expansion near the contours. 


\section{INTEGRAL EQUATIONS FOR USE IN DIRECT AND INVERSE PROBLEMS}

General solutions for plane elastic problems in terms of complex potentials (1) are given by the Kolosov-Muskhelishvili equations for the stress and displacement components in a Cartesian coordinate system $O x y$, as follows [1]:

$$
\begin{aligned}
& P \equiv \frac{\sigma_{x x}+\sigma_{y y}}{2}=\Phi(z)+\overline{\Phi(z)} \\
& D \equiv \frac{\sigma_{y y}-\sigma_{x x}}{2}+i \sigma_{x y}=\bar{z} \Phi^{\prime}(z)+\Psi(z) \\
& 2 G W \equiv 2 G\left(u_{x}+i u_{y}\right)=\kappa \phi(z)-z \overline{\Phi(z)}-\overline{\psi(z)} .
\end{aligned}
$$

Here, $\sigma_{x x}, \sigma_{y y}, \sigma_{x y}$ are stress components; $P$ is mean stress, $D$ is the complex stress deviator, $W=u_{x}+i u_{y}$ is complex displacement vector with the components $u_{x}, u_{y}$ along the $x$ and $y$ axes, respectively. Also, $G$ is the shear modulus, $\kappa=(3-4 v)$ for the plain stress and $\kappa=(3-v) /(1+v)$ for the plane stress conditions, where $v$ is Poisson's ratio, and $\Phi(z)=\phi^{\prime}(z), \Psi(z)=\psi^{\prime}(z)$ are complex potentials (holomorphic functions of a complex variable $\mathrm{z}=x+i y)$.

By making use of eqns (1)-(3), we find the following expressions for contour values of stress functions $P$ and $D$ :

$$
\begin{gathered}
2 P^{ \pm}= \pm\left(g+g^{\prime}\right)+\mathbf{S}(g)+\overline{\mathbf{S}(g)} \\
2 D^{ \pm}= \pm\left(h-e^{-2 i \vartheta} g\right)+\mathbf{S}\left(h-e^{-2 i \vartheta} g\right)-\mathbf{S}\left((\bar{t}-\bar{\zeta}) g^{\prime}\right)
\end{gathered}
$$

Here, $\vartheta$ is the angle between the positive direction of the real axis and the tangent to the contour. For the limiting values of the stress vector $\sigma(\zeta)=\sigma_{n}(\zeta)+i \sigma_{t}(\zeta)$ $\left(\sigma(\zeta)=P(\zeta)+e^{-2 i \vartheta(\zeta)} \overline{D(\zeta)}\right)$ and the tangential derivative of the displacement vectors $W^{\prime}(\zeta)=\kappa \Phi(\zeta)-\overline{\Phi(\zeta)}-e^{-2 i \vartheta(\zeta)}\left(\zeta \overline{\Phi^{\prime}(\zeta)}-\overline{\Psi(\zeta)}\right)$ one finds

$$
\begin{aligned}
2 \sigma^{ \pm} & = \pm\left(g+e^{-2 i \vartheta} h\right)+\mathbf{S}(g)+\overline{\mathbf{S}(g)}+e^{-2 i \vartheta} \overline{\mathbf{S}\left(h-e^{-2 i \vartheta} g\right)}-e^{-2 i \vartheta} \overline{\mathbf{S}\left((\bar{t}-\bar{\zeta}) g^{\prime}\right)} \\
2 W^{\prime \pm} & = \pm\left(\kappa g-e^{-2 i \vartheta} h\right)+\kappa \mathbf{S}(g)-\overline{\mathbf{S}(g)}+e^{-2 i \vartheta} \overline{\mathbf{S}\left(h-e^{-2 i \vartheta} g\right)}-e^{-2 i \vartheta} \overline{\mathbf{S}\left((\bar{t}-\bar{\zeta}) g^{\prime}\right)}
\end{aligned}
$$

Now we can derive the following CSIE for the first and the second fundamental problems of plane elasticity. Assuming that the known stress vector $N+i T$ is continuous across the contour, one finds $h=-e^{-2 i \vartheta} \bar{g}$ and thus, from eqn (10) we find:

$$
\mathbf{S}(g)+\overline{\mathbf{S}(g)}-e^{-2 i \vartheta} \overline{\mathbf{S}\left(e^{-2 i \vartheta}(g+\bar{g})\right)}-e^{-2 i \vartheta} \overline{\mathbf{S}\left((\bar{t}-\bar{\zeta}) g^{\prime}\right)}=2 N+2 i T
$$

In the second fundamental problem, we assumed that the known displacement vector $W_{x}^{\prime}+i W_{y}^{\prime}$ is continuous; thus, $\kappa g=e^{-2 i \vartheta} \bar{h}$ and so we get: 


$$
\kappa \mathbf{S}(g)-\overline{\mathbf{S}(g)}+e^{-2 i \vartheta} \overline{\mathbf{S}\left(e^{-2 i \vartheta}(\kappa \bar{g}-g)\right)}-e^{-2 i \vartheta} \overline{\mathbf{S}\left((\bar{t}-\bar{\zeta}) g^{\prime}\right)}=2 W_{X}^{\prime}+2 i W_{y}^{\prime}
$$

It can be shown that these CSIE coincide with previously published CSIE [3]. It is evident that both are of the first kind, so they can be solved by the method of mechanical quadratures outlined above. As a result, one finds the density $g(t)$, as well as $h(t)$. Thus, both potentials in eqn (1) become known. However, in order to analyse the stress-strain state in the entire domain, one should perform integration of the complex potentials, to obtain the stress functions and stress components, which implies integration at the nearcontour points.

Now let us discuss a previously-considered non-classical formulation [4]. The problem was formulated as follows: Find the complex potentials by using the data on the direction of the maximum principal stress (known angle $\theta_{j}$ counted from the real axis), at a number of discrete points $z_{j}(j=1 \ldots N)$, given inside the domain (these may also be on the boundary).

The principal directions are determined by the complex stress deviator as follows:

$$
D=\tau_{\max } e^{i \alpha}, \quad \tau_{\max }=|D|=\frac{\sigma_{1}-\sigma_{2}}{2} \geq 0, \quad \alpha=\arg (D)=\pi-2 \theta \quad(-\pi<\alpha \leq \pi) .
$$

Here, $\sigma_{1}$ and $\sigma_{2}$ are the principal stresses, $\theta$ is the direction of the major principal stress, $\sigma_{1}$, $\tau_{\max }$ is the maximum shear stresses. Bearing in mind that $\tau_{\max }$ is real, one can write:

$$
\operatorname{Im}\left[D(z, \bar{z}) e^{2 i \theta(z, \bar{z})}\right]=0 .
$$

So far as the principal directions are known at points $\mathrm{z}_{j}$ one arrives at the following optimisation problem:

$$
\sum_{j=1}^{N}\left\{\operatorname{Im}\left[\exp \left(-i \alpha_{j}\right)\left(\bar{z}_{j} \Phi^{\prime}\left(z_{j}\right)+\Psi\left(z_{j}\right)\right)\right]\right\}^{2} \Rightarrow \min .
$$

Let us seek the complex potentials in eqn (1), assuming continuity of the stress vector across the closed contour $\Gamma$ (similar to the first fundamental problem in eqn (12)), and introduce the real and imaginary parts of the sought function:

$$
\mu(t)=\operatorname{Re}(g(t)), \quad v(t)=\operatorname{Im}(g(t)) .
$$

Then, the complex potentials assume the following form:

$$
\Phi(z)=\frac{1}{2 \pi i} \int_{\Gamma} \frac{\mu(t)+i v(t)}{t-z} d t, \quad \Psi(z)=\frac{-1}{2 \pi i} \int_{\Gamma} \frac{2 \mu(t) e^{-2 i \vartheta(t)}+\bar{t}\left(\mu^{\prime}(t)+i v^{\prime}(t)\right)}{t-z} d t
$$

Thus, the expression for the complex stress deviator is:

$$
D(z, \bar{z})=\frac{-1}{\pi i} \int_{\Gamma} \frac{\mu(t) e^{-2 i \theta(t)}}{t-z} d t-\frac{1}{2 \pi i} \int_{\Gamma} \frac{\bar{t}-\bar{z}}{t-z}\left(\mu^{\prime}(t)+i v^{\prime}(t)\right) d t .
$$


Expressions (18) and (19) should satisfy the conditions of the single valuedness of the displacements:

$$
\int_{\Gamma}[\mu(t)+i v(t)] d t=0 .
$$

The boundary values of eqn (19) are to be found using eqns (2) and (3), as follows:

$$
D^{ \pm}=\mp e^{-2 i \vartheta_{\mu}}-\mathbf{S}\left(e^{-2 i \vartheta_{\mu}}\right)+\frac{1}{2} \mathbf{R}_{1}(\mu+i v)
$$

Here, the regular integral $\mathbf{R}_{1}$ appeared due to the second integral on the right-hand side of eqn (19), which is non-singular:

$$
\mathbf{R}_{1}(g)=-\mathbf{S}\left((\bar{t}-\bar{\zeta}) g^{\prime}\right)=-\frac{1}{\pi i} \int_{\Gamma} \frac{\bar{t}-\bar{\zeta}}{t-\zeta} g^{\prime}(t) d t=\frac{1}{\pi i} \int_{\Gamma}\left(\frac{d \bar{t} / d t}{t-\zeta}-\frac{\bar{t}-\bar{\zeta}}{(t-\zeta)^{2}}\right) g(t) d t
$$

In the optimisation problem (16), one must use the expression for the deviator in eqn (19) for internal points and in eqn (21) for boundary points. As it is evident from eqn (19) for the points located close to the contour, the first integral on the right-hand side is of a form similar to eqn (6). Calculation of the second integral does not present any numerical difficulties, because its kernel is $\exp [-2 \operatorname{iarg}(t-\zeta)]$, i.e., piecewise continuous and bounded.

Numerical approaches for solving eqn (16) can also be based on the method of mechanical quadratures, which implies numerical calculations of the integrals in eqns (19) and (21). If we assume that all data are internal, then the problem is reduced to a linear system of algebraic equations:

$$
\left\{\begin{array}{l}
\operatorname{Im}\left[\sum_{k=1}^{n} w_{k} \frac{2 \mu_{k} e^{-2 i \vartheta_{k}}-\left(e^{-2 i \vartheta_{k}}-e^{-2 i \arg \left(t_{k}-z_{j}\right)}\right)\left(\mu_{k}+i v_{k}\right)}{2 \pi i \exp \left(-2 i \theta_{j}\right)\left(t_{k}-z_{j}\right)}\right]=0, j=1 \ldots N, \\
\sum_{k=1}^{n} w_{k}\left(\mu_{k}+i v_{k}\right)=0 .
\end{array}\right.
$$

Here, $t_{k}$ and $w_{k}$ are, respectively, nodes and weights of a quadrature formula used for the discretisation, $\mu_{k}=\mu\left(t_{k}\right)$ and $v_{k}=v\left(t_{k}\right)$ are the sought real values of the density of the potential, while $\vartheta_{k}=\vartheta\left(t_{k}\right)$ are the slope angles at the nodes, $\theta_{j}$ are the known principal directions at the data points $\mathrm{z}_{j}$.

The system shown in eqn (23) consists of $\mathrm{N}+2$ real equations for the determination of $2 \mathrm{n}$ real unknowns. It is overspecified if $\mathrm{N}+2>2 \mathrm{n}$; and thus, should be solved approximately using the least squares method. We point out that for experimentally acquired data subject to measurement errors, the matrix of the system is affected by these errors. In the case when a part of the data is on the boundary, the first group of equations in eqn (23) should be modified by adding non-integral terms in eqn (21), which does not change its dimensions. 


\section{NUMERICAL APPROACH}

\subsection{Algorithm}

It is evident from the CSIE that we presented in the previous section, that a loss of accuracy in calculations of the complex potentials is observed for the Cauchy type integrals $\frac{1}{2 \pi i} \int_{\Gamma} \frac{g(t)}{t-z} d t$ (regardless of whether the contour is closed or open), when point $z$ is close to the contour. On the other hand, the singular integrals do not introduce any difficulties, even though in accordance with eqn (2), addition of one extra term is required. This allows us to employ Taylor's expansion of the complex potentials (1). As soon as eqn (1) presents two holomorphic functions which derivatives with respect to the conjugated variable, the Cauchy-Riemann conditions, vanish one can write:

$$
\Phi(z)=\Phi(\zeta)+\Phi^{\prime}(\zeta)(z-\zeta)+\overline{\bar{o}}(|z-\zeta|), \quad \zeta \in \Gamma .
$$

The derivative of the holomorphic function is found in the form [5]:

$$
\Phi^{\prime}(z)=\frac{1}{2 \pi i} \int_{\Gamma} \frac{g^{\prime}(t)}{t-z} d t .
$$

Note that this equation is valid for any smooth, closed contours, as well as for the open contours; provided that condition (20) is satisfied. Its boundary value can be found by the Plemelj-Sokhotski formulas as well as the boundary value of $\Phi(\zeta)$. Substitution of these boundary values into expression (24), leads to the following approximate equation:

$$
2 \Phi(z) \approx g(\zeta)+g^{\prime}(\zeta)(z-\zeta)+\mathbf{S}(g)+(z-\zeta) \mathbf{S}\left(g^{\prime}\right), \quad \zeta \in \Gamma .
$$

The proposed algorithm supposes that a specific data point $\mathrm{z}$ is supplied, and the equation for $\Gamma$ is known. It assumes the following simple steps:

1. Find the closest distance from the point to the contour.

2. If this distance is greater than a selected threshold, then perform integration based on the usual quadrature formulas for regular integrals.

3. If the minimum distance is smaller than the threshold, then find the distance from $z$ to the nearest node:

a) If this distance is small enough (below the threshold), then calculate the value of the complex potential by eqn (26), using the nearest node as the contour variable $\zeta$.

b) If the distance is greater than the selected threshold, then use an interpolation formula to determine $\zeta$ as the point closest to $z$, followed by calculations using eqn (26). Interpolation of the solution is also required, due to the non-integral terms present in eqn (26).

Note that if the contour is given by a set of ordered data points, then one may need to use an interpolation procedure. Polygonal approximation seems to be the simplest way, as it also allows one to find the slopes to the contour directly. More advanced approximations can be obtained by applying smoothing (e.g., by the fast Fourier transformation technique). 


\subsection{Example: Stresses near a rectilinear crack}

Let us consider the case of a rectilinear crack formed by a normal load, such that the density of the potential $\Phi(\mathrm{z})$ is bounded at the ends. For the sake of simplicity, let us assume that the potential is dimensionless (normalised by a characteristic value of applied load) and that the crack is of a unit half-length. Then it can be presented as follows:

$$
\Phi(z)=\Phi_{\text {sing }}(z)+\Phi_{\operatorname{regul}}(z), \quad \Phi_{\text {sing }}(z)=\frac{1}{2 \pi} \int_{-1}^{1} \frac{\sqrt{1-t^{2}}}{t-z} d t,
$$

Here, the term $\Phi_{\text {regul }}(z)$ on the right hand side remains regular, when $z$ tends to the contour. Therefore, we can analyse the first term $\Phi_{\text {sing }}(z)$ only.

Evaluation of the Cauchy integral gives an analytical solution for $\Phi_{\text {sing }}(z)$ in the form:

$$
\Phi_{\text {sing_anal }}(z)=\frac{1}{2}\left(\sqrt{z^{2}-1}-z\right) \text {. }
$$

Its derivative is:

$$
\Phi_{\text {sing_anal }}^{\prime}(z)=\frac{1}{2}\left(\frac{z}{\sqrt{z^{2}-1}}-1\right) .
$$

Therefore, Taylor's expansion for an analytical solution can be presented as:

$$
\Phi_{\text {sing_anal }}(z) \approx \frac{1}{2} \frac{\zeta(z-\zeta)}{\sqrt{\zeta^{2}-1}}+\frac{1}{2} \sqrt{\zeta^{2}-1}-\frac{z}{2}, \quad \zeta \in \Gamma,
$$

Numerical calculations of $\Phi_{\text {sing }}(\zeta)$ at the collocation points $\eta_{m}(m=1 \ldots n-1)$ produce:

$$
\begin{aligned}
& \Phi_{\text {sing_num }}\left(\eta_{m}\right)=\frac{1}{2 \pi} \int_{-1}^{1} \frac{\sqrt{1-t^{2}}}{t-\zeta} d t=\frac{1-\eta_{m}^{2}}{2 n} \sum_{k=1}^{n} \frac{1}{\xi_{k}-\eta_{m}}-\frac{\eta_{m}}{2}, \\
& \Phi_{\text {sing_num }}\left(\eta_{m}\right)=\frac{1}{2 \pi} \int_{-1}^{1} \frac{t}{\sqrt{1-t^{2}}\left(t-\eta_{m}\right)} d t=\frac{-1}{2 n} \sum_{k=1}^{n} \frac{\xi_{k}}{\xi_{k}-\eta_{m}} .
\end{aligned}
$$

For simplicity, let us assume that $\zeta=\eta m+i \varepsilon$, where $\varepsilon<<1$. Thus, eqn (26) assumes the form: 


$$
\begin{aligned}
\Phi_{\text {sing_num }}(\zeta) & =\frac{1-\eta_{m}^{2}}{2 n} \sum_{k=1}^{n} \frac{1}{\xi_{k}-\eta_{m}}-\frac{\eta_{m}}{2}+i \frac{\sqrt{1-\eta_{m}^{2}}}{2}+ \\
& +\left[\frac{1}{2 n} \sum_{k=1}^{n} \frac{\xi_{k}}{\xi_{k}-\eta_{m}}+\frac{i}{2} \frac{\eta_{m}}{\sqrt{1-\eta_{m}^{2}}}\right] \varepsilon \\
\Phi_{\text {sing_num }}(\zeta) & =\frac{1}{2 n} \sum_{k=1}^{n} \frac{1-\eta_{m}^{2}+\varepsilon \xi_{k}}{\xi_{k}-\eta_{m}}-\frac{\eta_{m}}{2}+\frac{i}{2} \frac{1-\eta_{m}^{2}+\varepsilon \eta_{m}}{\sqrt{1-\eta_{m}^{2}}} .
\end{aligned}
$$

In Table 1 we show the results of calculations using eqn (32) in comparison with the exact solution (28), analytical approximation (30) and calculations using the quadrature formula applied directly to eqn (27), for $n=8$ and $\varepsilon=0.5,0.05$, and 0.005 .

It is evident from Table 1 that analytical and numerical approximations (3rd and 4th columns) produce the same results, which can be explained by the high accuracy of the quadrature formula of the Chebyshev-Gauss type. The number of nodes $n=8$ is even redundant, but it is used here to compare the results at more points. For $\varepsilon=0.5$, the results

\begin{tabular}{|c|c|c|c|c|}
\hline $\begin{array}{c}\text { Point } \\
\text { coordinates }\end{array}$ & Exact (28) & $\begin{array}{c}\text { Analytic } \\
\text { approximation } \\
(30) \\
\end{array}$ & $\begin{array}{c}\text { Numerical } \\
\text { approximation } \\
(32)\end{array}$ & $\begin{array}{c}\text { Direct } \\
\text { calculations } \\
\text { of }(27) \\
\end{array}$ \\
\hline \multicolumn{5}{|l|}{$\varepsilon=0.5$} \\
\hline $0.924+0.5 \mathrm{i}$ & $-0.186+0.169 \mathrm{i}$ & $-0.212+0.795 \mathrm{i}$ & $-0.212+0.795 \mathrm{i}$ & $-0.186+0.169 \mathrm{i}$ \\
\hline $0.707+0.5 \mathrm{i}$ & $-0.166+0.222 \mathrm{i}$ & $-0.104+0.604 \mathrm{i}$ & $-0.104+0.604 \mathrm{i}$ & $-0.166+0.222 \mathrm{i}$ \\
\hline $0.383+0.5 \mathrm{i}$ & $-0.102+0.283 \mathrm{i}$ & $0.059+0.565 \mathrm{i}$ & $0.059+0.565 \mathrm{i}$ & $-0.102+0.283 \mathrm{i}$ \\
\hline $0.5 \mathrm{i}$ & $0.309 \mathrm{i}$ & $0.25+0.5 \mathrm{i}$ & $0.25+0.5 \mathrm{i}$ & $0.309 \mathrm{i}$ \\
\hline$-0.383+0.5 \mathrm{i}$ & $0.102+0.283 \mathrm{i}$ & $0.441+0.358 \mathrm{i}$ & $0.441+0.358 \mathrm{i}$ & $0.102+0.283 \mathrm{i}$ \\
\hline$-0.707+0.5 \mathrm{i}$ & $0.166+0.222 \mathrm{i}$ & $0.604+0.104 \mathrm{i}$ & $0.604+0.104 \mathrm{i}$ & $0.166+0.222 \mathrm{i}$ \\
\hline$-0.924+0.5 \mathrm{i}$ & $0.186+0.169 \mathrm{i}$ & $0.712-0.412 \mathrm{i}$ & $0.712-0.412 \mathrm{i}$ & $0.186+0.169 \mathrm{i}$ \\
\hline \multicolumn{5}{|l|}{$\varepsilon=0.05$} \\
\hline $0.924+0.05 \mathrm{i}$ & $-0.405+0.176 \mathrm{i}$ & $-0.437+0.252 \mathrm{i}$ & $-0.437+0.252 \mathrm{i}$ & $-0.43+0.133 \mathrm{i}$ \\
\hline $0.707+0.05 \mathrm{i}$ & $-0.329+0.33 \mathrm{i}$ & $-0.329+0.379 \mathrm{i}$ & $-0.329+0.379 \mathrm{i}$ & $-0.346+0.157 \mathrm{i}$ \\
\hline $0.383+0.05 \mathrm{i}$ & $-0.181+0.438 \mathrm{i}$ & $-0.166+0.472 \mathrm{i}$ & $-0.166+0.472 \mathrm{i}$ & $-0.189+0.164 \mathrm{i}$ \\
\hline $0.05 \mathrm{i}$ & $0.476 \mathrm{i}$ & $0.025+0.5 \mathrm{i}$ & $0.025+0.5 \mathrm{i}$ & $0.165 \mathrm{i}$ \\
\hline$-0.383+0.05 \mathrm{i}$ & $0.181+0.438 \mathrm{i}$ & $0.216+0.452 \mathrm{i}$ & $0.216+0.452 \mathrm{i}$ & $0.189+0.164 \mathrm{i}$ \\
\hline$-0.707+0.05 \mathrm{i}$ & $0.329+0.33 \mathrm{i}$ & $0.379+0.329 \mathrm{i}$ & $0.379+0.329 \mathrm{i}$ & $0.346+0.157 \mathrm{i}$ \\
\hline$-0.924+0.05 \mathrm{i}$ & $0.405+0.176 \mathrm{i}$ & $0.487+0.131 \mathrm{i}$ & $0.487+0.131 \mathrm{i}$ & $0.43+0.133 \mathrm{i}$ \\
\hline \multicolumn{5}{|l|}{$\varepsilon=0.005$} \\
\hline $0.924+5 \mathrm{E}-03 \mathrm{i}$ & $-0.456+0.189 \mathrm{i}$ & $-0.459+0.197 \mathrm{i}$ & $-0.459+0.197 \mathrm{i}$ & $-0.462+0.017 \mathrm{i}$ \\
\hline $0.707+5 \mathrm{E}-03 \mathrm{i}$ & $-0.351+0.351 \mathrm{i}$ & $-0.351+0.356 \mathrm{i}$ & $-0.351+0.356 \mathrm{i}$ & $-0.353+0.017 \mathrm{i}$ \\
\hline $0.383+5 \mathrm{E}-03 \mathrm{i}$ & $-0.19+0.459 \mathrm{i}$ & $-0.189+0.463 \mathrm{i}$ & $-0.189+0.463 \mathrm{i}$ & $-0.191+0.017 \mathrm{i}$ \\
\hline $5 \mathrm{E}-03 \mathrm{i}$ & $0.498 \mathrm{i}$ & $2.5 \mathrm{E}-03+0.5 \mathrm{i}$ & $2.5 \mathrm{E}-03+0.5 \mathrm{i}$ & $0.017 \mathrm{i}$ \\
\hline$-0.383+5 \mathrm{E}-03 \mathrm{i}$ & $0.19+0.459 \mathrm{i}$ & $0.194+0.461 \mathrm{i}$ & $0.194+0.461 \mathrm{i}$ & $0.191+0.017 \mathrm{i}$ \\
\hline$-0.707+5 \mathrm{E}-03 \mathrm{i}$ & $0.351+0.351 \mathrm{i}$ & $0.356+0.351 \mathrm{i}$ & $0.356+0.351 \mathrm{i}$ & $0.353+0.017 \mathrm{i}$ \\
\hline$-0.924+5 \mathrm{E}-03 \mathrm{i}$ & $0.456+0.189 \mathrm{i}$ & $0.464+0.185 \mathrm{i}$ & $0.464+0.185 \mathrm{i}$ & $0.462+0.017 \mathrm{i}$ \\
\hline
\end{tabular}
using Taylor's approximations are unsatisfactory, while exact and direct calculations

Table 1: Exact, analytical, numerical and direct calculations of the complex potential. 
coincide. For $\varepsilon=0.05$, all results are more or less of the same quality as exact solutions. For $\varepsilon=0.005$, calculations using direct Chebyshev integration of eqn (27) produce incorrect results, especially for the imaginary part of the complex potential $\Phi(\mathrm{z})$. In the meantime, Taylor's approximations (30) and (32) are remarkably close to calculations using the exact eqn (28).

\subsection{Example: Principal stress directions near a rectilinear crack}

We will use the results of the previous subsection to illustrate what are typical values of errors in the calculation of principal direction near a rectangular crack. For this purpose, let us select the derivative of the potential $\Phi(\mathrm{z})$ in the form similar to eqn (27):

$$
\Phi^{\prime}(z)=\Phi_{S}^{\prime}(z)+\Phi_{\mathrm{R}}^{\prime}(z), \quad \Phi_{S}^{\prime}(z)=\frac{1}{2 i \pi} \int_{-1}^{1} \frac{\sqrt{1-t^{2}}}{t-z} d t
$$

Here, $\Phi_{R}^{\prime}(z)$ is given by a regular integral, when $z$ tends to the contour point $\zeta$. We can still accept that the condition of single valuedness is satisfied, because the density of $\Phi(z)$ is odd and equal to $\frac{t}{2} \sqrt{1-t^{2}}+\arcsin t$.

For rectilinear boundaries, the second potential is $\Psi(z)=-z \Phi^{\prime}(z)$; therefore, $D(z, \bar{z})=(\bar{z}-z) \Phi^{\prime}(z)$ and the principal directions are determined by the argument of $\Phi^{\prime}(z)$ alone. Simplify by omitting the regular term in eqn (33), to find an exact expression for the principal directions:

$$
\theta(z)=-\frac{1}{2} \arg \left(\frac{-1}{\pi} \int_{-1}^{1} \frac{\sqrt{1-t^{2}}}{t-z} d t\right)=-\frac{1}{2} \arg \left(z-\sqrt{z^{2}-1}\right), \quad \operatorname{Im}(z)>0 .
$$

On the other hand, using Taylor's expansion for the function $\Phi_{S}^{\prime}(z)$ one can find an approximate expression for the principal directions at points $\eta_{m}+i \varepsilon$ :

$$
\theta_{\text {app }}\left(\eta_{m}+i \varepsilon\right) \approx \frac{-1}{2} \arg \left(\frac{\eta_{m}^{2}-1+i \varepsilon \eta m}{\sqrt{\eta_{m}^{2}-1}}-\eta_{m}-i \varepsilon\right), \quad m=1 \ldots n-1 .
$$

We can further use this equation only without referencing numerical calculations, because the results of both numerical and analytical calculations fully coincide, as was evident from the 3 rd and 4th columns of Table 1.

The results of calculations are summarised in Table 2 for $\varepsilon=0.5,0.05$, and 0.005 . It is evident from Table 2 that approximation (35) is incorrect for $\varepsilon=0.5$, while exact and direct calculations coincide. For $\varepsilon=0.05$, both approximate and direct calculations possess reasonable accuracy. For $\varepsilon=0.005$, the direct numerical calculations failed, while the approximation coincides with the exact calculations. 
Table 2: Comparisons of exact, approximate and direct calculations of the principal directions (degrees).

\begin{tabular}{|c|c|c|c|}
\hline Point coordinates & Exact (34) & Approximation (35) & $\begin{array}{l}\text { Direct calculations } \\
\text { from eqn (33) }\end{array}$ \\
\hline \multicolumn{4}{|l|}{$\varepsilon=0.5$} \\
\hline $0.924+0.5 \mathrm{i}$ & 21 & 38 & 21 \\
\hline $0.707+0.5 \mathrm{i}$ & 27 & 40 & 27 \\
\hline $0.383+0.5 \mathrm{i}$ & 35 & 48 & 35 \\
\hline $0.5 \mathrm{i}$ & 45 & 58 & 45 \\
\hline$-0.383+0.5 \mathrm{i}$ & 55 & 70 & 55 \\
\hline$-0.707+0.5 \mathrm{i}$ & 63 & 85 & 63 \\
\hline$-0.924+0.5 \mathrm{i}$ & 69 & -75 & 69 \\
\hline \multicolumn{4}{|l|}{$\varepsilon=0.05$} \\
\hline $0.924+0.05 \mathrm{i}$ & 12 & 15 & 9 \\
\hline $0.707+0.05 \mathrm{i}$ & 23 & 25 & 12 \\
\hline $0.383+0.05 \mathrm{i}$ & 34 & 35 & 20 \\
\hline $0.05 \mathrm{i}$ & 45 & 46 & 45 \\
\hline$-0.383+0.05 \mathrm{i}$ & 56 & 58 & 70 \\
\hline$-0.707+0.05 \mathrm{i}$ & 67 & 70 & 78 \\
\hline$-0.924+0.05 \mathrm{i}$ & 78 & 82 & 81 \\
\hline \multicolumn{4}{|l|}{$\varepsilon=0.005$} \\
\hline $0.924+5 \mathrm{E}-3 \mathrm{i}$ & 11 & 12 & 1 \\
\hline $0.707+5 \mathrm{E}-3 \mathrm{i}$ & 23 & 23 & 1 \\
\hline $0.383+5 \mathrm{E}-3 \mathrm{i}$ & 34 & 34 & 3 \\
\hline $5 \mathrm{E}-3 \mathrm{i}$ & 45 & 45 & 45 \\
\hline$-0.383+5 \mathrm{E}-3 \mathrm{i}$ & 56 & 56 & 87 \\
\hline$-0.707+5 \mathrm{E}-3 \mathrm{i}$ & 67 & 68 & 89 \\
\hline$-0.924+5 \mathrm{E}-3 \mathrm{i}$ & 79 & 79 & 89 \\
\hline
\end{tabular}

4 CONCLUSIONS

In this study, we investigated the applicability of Taylor's expansions and suggested an algorithm for the calculations of some characteristics of the stress state near the contour. We show that for small distances from the contour, direct application of quadrature formulas fails, while our proposed numerical approach produces highly accurate results.

\section{ACKNOWLEDGEMENT}

This work was partly supported by a grant from the Russian Foundation for Basic Research: grant number 20-05-00629 A.

\section{REFERENCES}

[1] Muskhelishvili, N.I., Some Basic Problems of the Mathematical Theory of Elasticity, P. Noordhoff: Groningen, the Netherlands, 1963.

[2] Savruk, M.P., Two-Dimensional Problems of Elasticity for Body with Cracks, Naukova Dumka: Kiev, Ukraine, 1981.

[3] Linkov, M., Boundary Integral Equations in Elasticity Theory, Kluwer, 2002. 
[4] Galybin, A.N. \& Mukhamediev, Sh.A., Determination of elastic stresses from discrete data on stress orientations. International Journal of Solids and Structures, 41(18-19), pp. 5125-5142, 2004.

[5] Gakhov, F.D., Boundary Value Problems, Dover Publications: New York, 1990. 